\title{
A NOTE ON STRONG SUMMABILITY OF TWO-DIMENSIONAL WALSH-FOURIER SERIES
}

\author{
G. TEPHNADZE
}

\begin{abstract}
In this paper we investigate strong summability of the two-dimensional WalshFourier series obtained in Weisz [18] (see Theorem W) and prove sharpness of this result.
\end{abstract}

2010 Mathematics Subject Classification. 42C10.

Key words and phrases: Walsh system, two-dimensional Walsh-Fourier series, Strong summability, martingale Hardy space.

Let $\mathbb{N}_{+}$denote the set of positive integers, $\mathbb{N}:=\mathbb{N}_{+} \cup\{0\}$. Denote by $Z_{2}$ the discrete cyclic group of order 2 , that is $Z_{2}=\{0,1\}$, where the group operation is the modulo 2 addition and every subset is open. The Haar measure on $Z_{2}$ is given such that the measure of a singleton is $1 / 2$. Let $G$ be the complete direct product of the countable infinite copies of the compact groups $Z_{2}$. The elements of $G$ are of the form $x=\left(x_{0}, x_{1}, \ldots, x_{k}, \ldots\right)$ with $x_{k} \in\{0,1\}(k \in \mathbb{N})$. The group operation on $G$ is the coordinate-wise addition, the measure (denote by $\mu$ ) and the topology are the product measure and topology of $Z_{2}=\{0,1\}$. The compact Abelian group $G$ is called the Walsh group. A base for the neighborhoods of $G$ can be given in the following way:

$$
\begin{aligned}
I_{0}(x): & =G, I_{n}(x):=I_{n}\left(x_{0}, \ldots, x_{n-1}\right) \\
: & =\left\{y \in G: y=\left(x_{0}, \ldots, x_{n-1}, y_{n}, y_{n+1}, \ldots\right)\right\},(x \in G, n \in \mathbb{N}) .
\end{aligned}
$$

These sets are called the dyadic intervals. Let $0=(0: i \in \mathbb{N}) \in G$ denote the null element of $G, I_{n}:=I_{n}(0)(n \in \mathbb{N})$. Set $e_{n}:=(0, \ldots, 0,1,0, \ldots) \in G$ the $n$th coordinate of which is 1 and the rest are zeros $(n \in \mathbb{N})$. Let $\bar{I}_{n}:=G \backslash I_{n}$.

If $n \in \mathbb{N}$, then $n=\sum_{i=0}^{\infty} n_{i} 2^{i}$, where $n_{i} \in\{0,1\} \quad(i \in \mathbb{N})$, i.e. $n$ is expressed in the number system of base 2. Denote $|n|:=\max \left\{j \in \mathbb{N}: n_{j} \neq 0\right\}$, that is, $2^{|n|} \leq n<2^{|n|+1}$.

It is easy to show that for every odd number $n_{0}=1$ and we can write $n=1+\sum_{i=1}^{|n|} n_{j} 2^{i}$, where $n_{j} \in\{0,1\}, \quad\left(j \in \mathbb{N}_{+}\right)$.

For $k \in \mathbb{N}$ and $x \in G$ let as denote by

$$
r_{k}(x):=(-1)^{x_{k}} \quad(x \in G, k \in \mathbb{N})
$$

the $k$-th Rademacher function.

The Walsh-Paley system is defined as the sequence of Walsh-Paley functions:

$$
w_{n}(x):=\prod_{k=0}^{\infty}\left(r_{k}(x)\right)^{n_{k}}=r_{|n|}(x)(-1) \sum_{k=0}^{|n|-1} n_{k} x_{k} \quad\left(x \in G, n \in \mathbb{N}_{+}\right)
$$

The research was supported by Shota Rustaveli National Science Foundation grant YS-18-043. 
The Walsh-Dirichlet kernel is defined by

$$
D_{n}(x)=\sum_{k=0}^{n-1} w_{k}(x)
$$

Recall that (see [8, p. 7])

$$
D_{2^{n}}(x)=\left\{\begin{array}{cc}
2^{n}, & x \in I_{n} \\
0, & x \in \bar{I}_{n}
\end{array}\right.
$$

Let $n \in \mathbb{N}$ and $n=\sum_{i=0}^{\infty} n_{i} 2^{i}$. Then

$$
D_{n}(x)=w_{n}(x) \sum_{j=0}^{\infty} n_{j} w_{2^{j}}(x) D_{2^{j}}(x) .
$$

Set $G^{2}:=G \times G$. The rectangular partial sums $S_{M, N}$ of the 2-dimensional Walsh-Fourier series of a function $f \in L_{2}\left(G^{2}\right)$ are defined as follows:

$$
S_{M, N} f(x, y):=\sum_{i=0}^{M-1} \sum_{j=0}^{N-1} \widehat{f}(i, j) w_{i}(x) w_{j}(y)
$$

where the number

$$
\widehat{f}(i, j)=\int_{G^{2}} f(x, y) w_{i}(x) w_{j}(y) d \mu(x, y)
$$

is said to be the $(i, j)$-th Walsh-Fourier coefficient of the function $f$.

The norms (or quasi-norms) of the spaces $L_{p}\left(G^{2}\right)$ and weak $-L_{p}\left(G^{2}\right)$ are respectively defined by

$$
\|f\|_{p}:=\left(\int_{G^{2}}|f|^{p} d \mu\right)^{1 / p}, \quad\|f\|_{\text {weak }-L_{p}\left(G^{2}\right)}:=\sup _{\lambda>0} \lambda \mu(f>\lambda)^{1 / p} \quad(0<p<\infty) .
$$

The $\sigma$-algebra generated by the dyadic 2-dimensional $I_{n}(x) \times I_{n}(y)$ square of measure $2^{-n} \times 2^{-n}$ will be denoted by $\digamma_{n}(n \in \mathbb{N})$. Denote by $f=\left(f_{n}, n \in \mathbb{N}\right)$ the one-parameter martingale with respect to $\digamma_{n}(n \in \mathbb{N})$, (for details see e.g. Weisz [16] and [17]). The maximal function of a martingale $f$ is defined by

$$
f^{*}=\sup _{n \in \mathbb{N}}\left|f_{n}\right| .
$$

Let $f \in L_{1}\left(G^{2}\right)$. Then the dyadic maximal function is given by

$$
f^{*}(x, y)=\sup _{n \in \mathbb{N}} \frac{1}{\mu\left(I_{n}(x) \times I_{n}(y)\right)}\left|\int_{I_{n}(x) \times I_{n}(y)} f(s, t) d \mu(s, t)\right|, \quad(x, y) \in G^{2} .
$$

The dyadic Hardy martingale space $H_{p}\left(G^{2}\right)(0<p<\infty)$ consists of all functions $f$ for which

$$
\|f\|_{H_{p}\left(G^{2}\right)}:=\left\|f^{*}\right\|_{p}<\infty
$$


If $f \in L_{1}\left(G^{2}\right)$, then it is easy to show that the sequence $\left(S_{2^{n}, 2^{n}} f: n \in \mathbb{N}\right)$ is a martingale. If $f=\left(f_{n}, n \in \mathbb{N}\right)$ is a martingale, then the Walsh-Fourier coefficients must be defined in a slightly different manner:

$$
\widehat{f}(i, j):=\lim _{k \rightarrow \infty} \int_{G} f_{k}(x, y) w_{i}(x) w_{j}(y) d \mu(x, y) .
$$

The Walsh-Fourier coefficients of $f \in L_{1}\left(G^{2}\right)$ are the same as those of the martingale $\left(S_{2^{n}, 2^{n}} f: n \in \mathbb{N}\right)$ obtained from $f$.

A bounded measurable function $a$ is a p-atom, if there exists a dyadic 2-dimensional cube $I \times I$, such that

$$
\int_{I \times I} a d \mu=0, \quad\|a\|_{\infty} \leq \mu(I \times I)^{-1 / p}, \quad \operatorname{supp}(a) \subset I \times I .
$$

The dyadic Hardy martingale spaces $H_{p}\left(G^{2}\right)$ for $0<p \leq 1$ have atomic characterizations (for details see e.g. Weisz [16] and [17]).

Lemma 1. A martingale $f=\left(f_{n}: n \in \mathbb{N}\right)$ is in $H_{p}\left(G^{2}\right)(0<p \leq 1)$ if and only if there exist a sequence $\left(a_{k}, k \in \mathbb{N}\right)$ of $p$-atoms and a sequence $\left(\mu_{k}, k \in \mathbb{N}\right)$ of a real numbers such that

$$
\sum_{k=0}^{\infty} \mu_{k} S_{2^{n}, 2^{n}} a_{k}=f_{n}
$$

and

$$
\sum_{k=0}^{\infty}\left|\mu_{k}\right|^{p}<\infty
$$

Moreover,

$$
\|f\|_{H_{p}} \backsim \inf \left(\sum_{k=0}^{\infty}\left|\mu_{k}\right|^{p}\right)^{1 / p}
$$

where the infimum is taken over all decomposition of $f$ of the form (3).

It is known [7, p. 125] that the Walsh-Paley system is not a Schauder basis in $L_{1}\left(G^{2}\right)$. Moreover, (for details see [8]) there exists a function in the dyadic martingale Hardy space $H_{p}\left(G^{2}\right)(0<p \leq 1)$ for which the partial sums are not bounded in $L_{p}\left(G^{2}\right)$. However, Weisz [18] proved the following estimation:

Theorem W. Let $\alpha \geq 0,0<p \leq 1$ and $f \in H_{p}\left(G^{2}\right)$. Then there exists an absolute constant $c_{p}$, depending only on $p$, such that

$$
\sup _{n, m \geq 2}\left(\frac{1}{\log n \log m}\right)^{[p]} \sum_{2^{-\alpha} \leq k / l \leq 2^{\alpha},(k, l) \leq(n, m)} \frac{\left\|S_{k, l} f\right\|_{p}^{p}}{(k l)^{2-p}} \leq c_{p}\|f\|_{H_{p}}^{p},
$$

where $[p]$ denotes the integer part of $p$.

In the case when $\alpha=0$ and $p=1$ from Theorem $\mathrm{W}$ we obtain that the following is true:

Theorem W1. Let $f \in H_{1}\left(G^{2}\right)$. Then there exists an absolute constant $c$, such that

$$
\frac{1}{\log ^{2} n} \sum_{k=0}^{n} \frac{\left\|S_{k, k} f\right\|_{1}}{k^{2}} \leq c\|f\|_{H_{1}\left(G^{2}\right)} \text {. }
$$


When $\alpha=0$ and $0<p<1$ from Theorem $\mathrm{W}$ follows the following result:

Theorem W2. Let $0<p<1$ and $f \in H_{p}\left(G^{2}\right)$. Then there exists an absolute constant $c_{p}$, depending only on $p$, such that

$$
\sum_{k=0}^{\infty} \frac{\left\|S_{k, k} f\right\|_{p}^{p}}{k^{4-2 p}} \leq c_{p}\|f\|_{H_{p}\left(G^{2}\right)}^{p} .
$$

Goginava and Gogoladze [3] generalized the estimate (4) (for details see [5] and [1]) and proved that for any $f \in H_{1}\left(G^{2}\right)$, there exists an absolute constant $c$, such that

$$
\sum_{n=1}^{\infty} \frac{\left\|S_{n, n} f\right\|_{1}}{n \log ^{2} n} \leq c\|f\|_{H_{1}\left(G^{2}\right)}
$$

The estimate (5) was generalized in [11] and it was proved that for any $0<p<1$ and $f \in H_{p}\left(G^{2}\right)$, there exists an absolute constant $c_{p}$, depending only on $p$, such that

$$
\sum_{n=1}^{\infty} \frac{\left\|S_{n, n} f\right\|_{p}^{p}}{n^{3-2 p}} \leq c_{p}\|f\|_{H_{p}\left(G^{2}\right)}^{p} .
$$

In [10] and [11] it was proved that the following is true:

Theorem T1. Let $0<p \leq 1$ and $\Phi: \mathbb{N} \rightarrow[1, \infty)$ be any nondecreasing function, satisfying the condition $\lim _{n \rightarrow \infty} \Phi(n)=+\infty$. Then there exists a martingale $f \in H_{p}\left(G^{2}\right)$ such that

$$
\sum_{n=1}^{\infty} \frac{\left\|S_{n, n} f\right\|_{w e a k-L_{p}}^{p} \Phi(n)}{n^{3-2 p} \log ^{2[p]} n}=\infty
$$

where $[p]$ denotes the integer part of $p$.

Analogical problems for the one-dimensional case were proved in [1], [2], [9], [12], [13], [15] and for the two-dimensional case in [5], [6], [7].

In view of Theorem $\mathrm{T} 1$ we can conclude that the sequence $\left\{1 /\left(n^{3-2 p} \log ^{2[p]} n\right)\right\}_{n=1}^{\infty}$ in inequalities (6) and (7) can not be improved, which gives sharpness for $\alpha=0$.

In this paper we consider the analogous problem for $\alpha>0$ and prove the sharpness of the sequence in Theorem $\mathrm{W}$ when $\alpha>0$ :

Theorem 1. Let $0<p<1, \alpha>0$ and $\Phi: \mathbb{N}^{2} \rightarrow[1, \infty)$ satisfies the conditions

$$
\Phi\left(m_{1}, n_{1}\right) \geq \Phi\left(m_{2}, n_{2}\right) \text { when } m_{1} \geq m_{2} \text { and } n_{1} \geq n_{2}
$$

and

$$
\lim _{m, n \rightarrow \infty} \Phi(m, n)=+\infty .
$$

Then there exists a martingale $f \in H_{p}\left(G^{2}\right)$ such that

$$
\sup _{n, m \in \mathbb{N}_{+} 2^{-\alpha} \leq k / l \leq 2^{\alpha},(k, l) \leq(n, m)} \sum_{(k l)^{2-p}} \frac{\left\|S_{k, l} f\right\|_{p}^{p} \Phi(k, l)}{(k .}
$$


Proof. Under the condition (9) there exists an increasing sequence of positive integers $\left\{\alpha_{k}: k \geq 0\right\}$ such that $\alpha_{0} \geq 2, \alpha_{k}+[\alpha]+1<\alpha_{k+1}$ and

$$
\sum_{k=0}^{\infty} \Phi^{-p / 4}\left(2^{\alpha_{k}}, 2^{\alpha_{k}}\right)<\infty
$$

Let

$$
f_{n}=\sum_{\left\{k ; \alpha_{k}+[\alpha]+1<n\right\}} \lambda_{k} a_{k}
$$

where

$$
\lambda_{k}:=2^{2[\alpha]+2} \Phi^{-1 / 4}\left(2^{\alpha_{k}}, 2^{\alpha_{k}}\right)
$$

and

$$
a_{k}(x, y):=2^{\alpha_{k}(2 / p-2)-2[\alpha]-2}\left(D_{2^{\alpha_{k}+[\alpha]+1}}(x)-D_{2^{\alpha_{k}}}(x)\right)\left(D_{2^{\alpha_{k}+[\alpha]+1}}(y)-D_{2^{\alpha_{k}}}(y)\right) .
$$

Since

$$
\begin{aligned}
& S_{2^{n}, 2^{n}} a_{k}=\left\{\begin{array}{l}
a_{k}, \quad \alpha_{k}+[\alpha]+1<n, \\
0, \quad \alpha_{k}+[\alpha]+1 \geq n,
\end{array}\right. \\
& \operatorname{supp}\left(a_{k}\right)=I_{\alpha_{k}}^{2}, \quad \int_{I_{\alpha_{k}}^{2}} a_{k} d \mu=0, \quad\left\|a_{k}\right\|_{\infty} \leq 2^{2 \alpha_{k} / p}=\left(\mu\left(\operatorname{supp} a_{k}\right)\right)^{-1 / p}
\end{aligned}
$$

from Lemma 1 and (10) we obtain that $f \in H_{p}\left(G^{2}\right)$.

It is obvious that

$$
=\left\{\begin{array}{l}
\frac{2^{\alpha_{k}(2 / p-2)}}{\Phi^{1 / 4}\left(2^{\left.\alpha_{k}, 2^{\alpha}\right)}\right)}, \quad \text { if }(i, j) \in\left\{2^{\alpha_{k}}, \ldots, 2^{\alpha_{k}+[\alpha]+1}-1\right\} \times\left\{2^{\alpha_{k}}, \ldots, 2^{\alpha_{k}+[\alpha]+1}-1\right\}, k \in \mathbb{N} \\
0, \quad \text { if }(i, j) \notin \bigcup_{k=1}^{\infty}\left\{2^{\alpha_{k}}, \ldots, 2^{\alpha_{k}+[\alpha]+1}-1\right\} \times\left\{2^{\alpha_{k}}, \ldots, 2^{\alpha_{k}+[\alpha]+1}-1\right\} .
\end{array}\right.
$$

Let $2^{\alpha_{k}}<m, n<2^{\alpha_{k}+[\alpha]+1}$. In view of (11) we can conclude that

$$
\begin{aligned}
& S_{m, n} f(x, y) \\
& =\sum_{\eta=0}^{k-1} \sum_{i=2^{\alpha_{\eta}}}^{2^{\alpha_{\eta}+1}} \sum_{j=2^{\alpha_{\eta}}}^{2^{\alpha_{\eta}+1}-1} \widehat{f}(i, j) w_{i}(x) w_{j}(y)+\sum_{i=2^{\alpha_{k}}}^{m-1} \sum_{j=2^{\alpha_{k}}}^{n-1} \widehat{f}(i, j) w_{i}(x) w_{j}(y) \\
& =\sum_{\eta=0}^{k-1} \sum_{i=2^{\alpha_{\eta}}}^{2^{\alpha_{\eta}+1}-1} \sum_{j=2^{\alpha_{\eta}}} \frac{2^{\alpha_{\eta}(2 / p-2)} w_{i}(x) w_{j}(y)}{\Phi^{1 / 4}\left(2^{\alpha_{\eta}}, 2^{\alpha_{\eta}}\right)}+\sum_{i=2^{\alpha_{k}}}^{m-1} \sum_{j=2^{\alpha_{k}}}^{n-1} \frac{2^{\alpha_{k}(2 / p-2)} w_{i}(x) w_{j}(y)}{\Phi^{1 / 4}\left(2^{\alpha_{k}}, 2^{\alpha_{k}}\right)} \\
& =\sum_{\eta=0}^{k-1} \frac{2^{\alpha_{\eta}(2 / p-2)}}{\Phi^{1 / 4}\left(2^{\alpha_{\eta}}, 2^{\alpha_{\eta}}\right)}\left(D_{2^{\alpha_{\eta}+1}}(x)-D_{2^{\alpha_{\eta}}}(x)\right)\left(D_{2^{\alpha_{\eta}+1}}(y)-D_{2^{\alpha_{\eta}}}(y)\right) \\
& +\frac{2^{\alpha_{k}(2 / p-2)}}{\Phi^{1 / 4}\left(2^{\alpha_{k}}, 2^{\alpha_{k}}\right)}\left(D_{m}(x)-D_{2^{\alpha_{k}}}(x)\right)\left(D_{n}(y)-D_{2^{\alpha_{k}}}(y)\right) \\
& :=I+I I \text {. }
\end{aligned}
$$


Let $(x, y) \in\left(G \backslash I_{1}\right) \times\left(G \backslash I_{1}\right), n$ and $m$ are odd numbers, such that $2^{\alpha_{k}}<m, n<2^{\alpha_{k}+[\alpha]+1}$. Since $\alpha_{k} \geq 2(k \in \mathbb{N})$, if we combine (11) and (2) it follows that

$$
D_{2^{\alpha_{k}}}(x)=D_{2^{\alpha_{k}}}(y)=0
$$

and

$$
|I I|=\frac{2^{\alpha_{k}(2 / p-2)}}{\Phi^{1 / 4}\left(2^{\alpha_{k}}, 2^{\alpha_{k}}\right)}\left|w_{m}(x) w_{1}(x) D_{1}(x) w_{n}(y) D_{1}(y)\right|=\frac{2^{\alpha_{k}(2 / p-2)}}{\Phi^{1 / 4}\left(2^{\alpha_{k}}, 2^{\alpha_{k}}\right)} .
$$

By applying (1) and the condition $\alpha_{n} \geq 2(n \in \mathbb{N})$ for $I$ we have that

$$
I=\sum_{\eta=0}^{k-1} \frac{2^{\alpha_{\eta}(2 / p-2)}}{\Phi^{1 / 4}\left(2^{\alpha_{\eta}}\right)}\left(D_{2^{\alpha_{\eta}+1}}(x)-D_{2^{\alpha_{\eta}}}(x)\right)\left(D_{2^{\alpha_{\eta}+1}}(y)-D_{2^{\alpha_{\eta}}}(y)\right)=0 .
$$

By combining (13) and (14), for $2^{\alpha_{k}}<m, n<2^{\alpha_{k}+[\alpha]+1}$ we get that

$$
\begin{aligned}
& \geq \frac{2^{\alpha_{k}(2 / p-2)}}{2 \Phi^{1 / 4}\left(2^{\alpha_{k}}, 2^{\alpha_{k}}\right)}\left(\mu\left\{(x, y) \in\left(G \backslash I_{1}\right) \times\left(G \backslash I_{1}\right):\left|S_{m, n} f(x, y)\right| \geq \frac{2^{\alpha_{k}(2 / p-2)}}{2 \Phi^{1 / 4}\left(2^{\alpha_{k}}, 2^{\alpha_{k}}\right)}\right\}\right)^{1 / p} \\
& \geq \frac{2^{\alpha_{k}(2 / p-2)}}{2 \Phi^{1 / 4}\left(2^{\alpha_{k}}, 2^{\alpha_{k}}\right)} \mu\left(\left(G \backslash I_{1}\right) \times\left(G \backslash I_{1}\right)\right) \geq \frac{c_{p} 2^{\alpha_{k}(2 / p-2)}}{\Phi^{1 / 4}\left(2^{\alpha_{k}}, 2^{\alpha_{k}}\right)} .
\end{aligned}
$$

According to (8), (9) and (15) we can conclude that

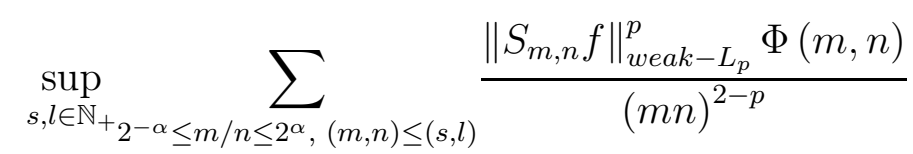

$$
\begin{aligned}
& \geq \sum_{2^{\alpha_{k}<m, n \leq 2^{\alpha_{k}+\alpha}}} \frac{\left\|S_{m, n} f\right\|_{\text {weak-L }}^{p} \Phi(m, n)}{(m n)^{2-p}} \\
& \geq \frac{c_{p} \Phi\left(2^{\alpha_{k}}, 2^{\alpha_{k}}\right)}{2^{\alpha_{k}(4-2 p)}} \sum_{2^{\alpha_{k}<m, n \leq 2^{\alpha_{k}+\alpha}}}\left\|S_{m, n} f\right\|_{\text {weak-L } L_{p}}^{p} \\
& \geq \frac{c_{p} \Phi\left(2^{\alpha_{k}}, 2^{\alpha_{k}}\right)}{2^{\alpha_{k}(4-2 p)}} \sum_{2^{\alpha_{k}-1}<m, n \leq 2^{\alpha_{k}-1+\alpha}}\left\|S_{2 m+1,2 n+1} f\right\|_{\text {weak-L }}^{p} \\
& \geq \frac{c_{p} \Phi\left(2^{\alpha_{k}}, 2^{\alpha_{k}}\right)}{2^{\alpha_{k}(4-2 p)}} \frac{2^{\alpha_{k}(2-2 p)}}{\Phi^{1 / 4}\left(2^{\alpha_{k}}, 2^{\alpha_{k}}\right)} \sum_{2^{\alpha_{k}-1}<m, n \leq 2^{\alpha_{k}-1+\alpha}} 1 \\
& \geq \frac{c_{p} \Phi^{3 / 4}\left(2^{\alpha_{k}}, 2^{\alpha_{k}}\right)}{2^{2 \alpha_{k}}}\left(2^{\alpha_{k}-1+\alpha}-2^{\alpha_{k}-1}-1\right)^{2} \\
& \geq c_{p} \Phi^{3 / 4}\left(2^{\alpha_{k}}, 2^{\alpha_{k}}\right) \rightarrow \infty, \quad \text { as } \quad k \rightarrow \infty .
\end{aligned}
$$

By combining (10,16) we complete the proof of Theorem 1. 


\section{REFERENCES}

[1] I. Blahota, K. Nagy, L. E. Persson, G. Tephnadze, A sharp boundedness result concerning some maximal operators of partial sums with respect to Vilenkin systems, Georgian Math., J., DOI: https://doi.org/10.1515/gmj-2018-0045.

[2] G. Gàt, Investigations of certain operators with respect to the Vilenkin system, Acta Math. Hungar. 61 (1993), no. 1-2, 131-149.

[3] U. Goginava, L. D. Gogoladze, Strong convergence of cubic partial sums of two-dimensional WalshFourier series, Constructive theory of functions, 108-117, Prof. M. Drinov Acad. Publ. House, Sofia, 2012.

[4] B. Golubov, A. Efimov, V. Skvortsov, Walsh series and transformations. Theory and applications. Translated from the 1987 Russian original in Mathematics and its Applications (Soviet series) 64, Kluwer Academic Publishers Group, Dordrecht, 1991.

[5] N. Memić, I. Simon, G. Tephnadze, Strong convergence of two-dimensional Vilenkin-Fourier series, Math. Nachr., 289, (2016), No. 4, 485-500.

[6] K. Nagy, Approximation by Nörlund means of Walsh-Kaczmarz-Fourier series, Georgian Math. J. 18 (2011), no. 1, 147-162.

[7] L. E. Persson, G. Tephnadze, P. Wall, On an approximation of two-dimensional Walsh-Fourier series in the martingale Hardy spaces, Ann. Funct. Anal., 9, 1 (2018), 137-150.

[8] F. Schipp, W.R. Wade, P. Simon, Walsh Series. An introduction to dyadic harmonic analysis, with the collabotation with J. Pál, Adam Hilger, Bristol, 1990.

[9] P. Simon, Strong convergence theorem for Vilenkin-Fourier series, J. Math. Anal. Appl. 245 (2000), no. $1,52-68$.

[10] G. Tephnadze, A note on the strong convergence of two-dimensional Walsh-Fourier series, Proc. A. Razmadze Math. Inst., 162 (2013), 93-97.

[11] G. Tephnadze, Strong convergence of two-dimensional Walsh-Fourier series, Ukrainian Math. J., 65, (2013), No. 6, 914-927.

[12] G. Tephnadze, On the convergence of partial sums with respect to Vilenkin system on the martingale Hardy spaces, J. Contemp. Math. Anal., 53, 5, (2018) 294-306.

[13] G. Tephnadze, On the partial sums of Walsh-Fourier series, Colloq. Math., 141, 2 (2015), 227-242.

[14] G. Tutberidze, A note on the strong convergence of partial sums with respect to Vilenkin system, J. Contemp. Math. Anal., (to appear).

[15] G. Tutberidze, Modulus of continuity and boundedness of subsequences of Vilenkin-Fejér means in the martingale Hardy spaces, Georgian Math. J., (to appear).

[16] F. Weisz, Martingale Hardy spaces and their applications in Fourier analysis, Lecture Notes in Mathematics, 1568, Springer-Verlag, Berlin, 1994.

[17] F. Weisz, Summability of multi-dimensional Fourier series and Hardy spaces, Kluwer Academic Publishers, Dordrecht, 2002.

[18] F. Weisz, Strong convergence theorems for two-parameter Walsh-Fourier and trigonometric-Fourier series. (English) Studia Math. 117, (1996), No.2, 173-194.

George Tephnadze, The University of Georgia, School of Science and Technology, 77a Merab Kostava St, Tbilisi, 0128, Georgia.

E-mail address: g.tephnadze@ug.edu.ge 